M. MAGISTRINI

INRA-Haras

Nati onaux, PRMD,

Equi pe Reproducti on Equine, 37380 Nouzi lly

email : michele.magi strini@ tours.inra.fr

\title{
La qualité des spermatozoïdes et du plasma séminal : quelles sont les fonctions explorables?
}

Actuellement la méthode de routine pour évaluer la fertilité potentielle d'un étalon est la réalisation d'un spermogramme (collecte de 5 éjaculats à $24 \mathrm{~h}$ d'intervalle) au cours duquel des paramètres séminaux quantitatifs (volume, concentration et nombre total en spermatozoïdes, pH) et qualitatifs (pourcentages de spermatozoïdes mobiles, vivants, de formes anormales) sont analysés. Cependant certains étalons, dont les paramètres séminaux sont supérieurs ou égaux aux seuils définis, ont une fertilité réduite. D'autre part, après conservation de la semence à l'état frais ou congelé, on observe parfois des résultats incohérents entre la mobilité des spermatozoïdes et la fertilité. II semble donc indispensable d'étudier d'autres critères afin d'expliquer et/ou de pallier ces problèmes de subfertilité ou d'infertilité. De plus I'utilisation de critères permettant d'évaluer d'autres fonctions que la mobilité pourrait nous permettre de mettre en évidence et d'expliquer des différences entre techniques de conservation.

Les spermatozoïdes sont des cellules complexes qui doivent maintenir l'intégrité de toutes ou partie de leurs fonctions pour être fécondants. Sur le tableau 1 sont présentés les différents compartiments de la cellule et les fonctions qui y sont associées. Pour évaluer ces fonctions, des tests ont été mis au point et sont développés chez les équins. Malheureusement ils n'ont pas pour le moment dépassé les limites du laboratoire. La plupart de ces méthodes font appel à la microscopie à fluorescence et parfois à la cytométrie en flux. Cette technique permet d'analyser un nombre très élevé de cellules en un temps très court.

\section{Les spermatozoïdes}

\section{La tête du spermatozoïde}

\section{La membrane plasmi que}

Pour pouvoir se fixer à la zone pellucide de l'ovocyte, le spermatozoïde doit avoir une membrane plasmique intègre. Pour évaluer cette intégrité, la coloration à l'éosine-nigrosine a été largement développée dans les différentes espèces de mammifères domestiques. Plus récemment de nouvelles méthodes ont été mises au point.

\section{Sondes de vitalité}

Le kit "Fertilight" " est composé de 2 sondes fluorescentes qui se fixent sur I'ADN du noyau : Ie SYBR-14 colore en vert le noyau de toutes les cellules et l'iodure de propidium ne pénètre que dans les cellules dont la membrane est lésée. Ainsi des spermatozoïdes vivants émettront une fluorescence verte et les spermatozoïdes morts une fluorescence rouge; il peut arriver que certains spermatozoïdes présentent les deux colorations; ils sont alors classés dans une 3ème catégorie : les " moribonds ". Ces sondes peuvent être analysées en microscopie à fluorescence, mais aussi en cytométrie en flux. D'autres association de sondes ont été utilisées pour évaluer la vitalité des spermatozoïdes : la carboxyfluorescéine (CFDA) ou ses dérivés, associée à l'iodure de propidium, la calcéineAM associée à l'éthidium homodimère-1.

Test de fixation à la zone pellucide

II est indispensable de disposer d'ovocytes équins en grande quantité, ce qui limite l'utilisation de cette methode. Pour pallier ce pro-

Tableau 1. Fonctions cellulaires du spermatozoïde et méthodes d'analyse.

\begin{tabular}{|l|l|}
\hline $\begin{array}{l}\text { Compartiments cellulaires } \\
\text { et fonctions analysées }\end{array}$ & Méthodes d'analyse \\
\hline $\begin{array}{l}\text { Tête du spermatozoïde } \\
\text { Intégrité de la membrane plasmique } \\
\text { Intégrité de l'acrosome }\end{array}$ & $\begin{array}{l}\text { Sondes fluorescentes : SYBR-14 et lodure de propidium } \\
\text { Lectine (agglutinine de Pi sum sati vum) } \\
\text { + marqueur fluorescent (isothiocyanate de fluorescéine) } \\
\text { Sonde fluorescente : acridine orange }\end{array}$ \\
\hline Intégrité de l'ADN & $\begin{array}{l}\text { Evaluation de l'ATP : dosage ou sonde fluorescente (Rhodamine 123) } \\
\text { Flagelle }\end{array}$ \\
$\begin{array}{l}\text { Intégrité de la membo-osmotique } \\
\text { Intégrité de l'activité du flagelle }\end{array}$ & $\begin{array}{l}\text { Analyse automatisée de la mobilité : } \\
\text { CASA (Computer Assisted Sperm Analysis) }\end{array}$ \\
\hline
\end{tabular}


blème, les travaux ont été réalisés sur des ovocytes stockés dans des solutions hypersalines. Ils ont montré une relation entre le nombre de spermatozoïdes fixés à la zone pellucide et la fertilité des étalons. Cependant ce test est lourd à mettre en place et il est utilisé de façon ponctuelle dans peu de laboratoires.

\section{L'acrosome}

La vésicule acrosomique recouvre le noyau du spermatozoïde et se met en place au cours de la spermiogenèse dans le testicule. Après sa fixation à l'ovocyte, le spermatozoïde doit traverser la zone pellucide et pour cela, il doit faire " la réaction acrosomique ". Cette réaction consiste en la fusion entre la membrane plasmique et la membrane acrosomique externe qui va libérer les enzymes nécessaires au passage de la zone pellucide. De nombreuses colorations ont été décrites dans la littérature et, depuis quelques années, I'utilisation d'une lectine, l'agglutinine de Pisum Sati vum, couplée à un marqueur fluorescent, l'isothiocyanate de fluorescéine, a été développée.

Après perméabilisation de la membrane plasmique, cette lectine se fixe au contenu de l'acrosome et met ainsi en évidence la présence ou l'absence de la vésicule acrosomique. Cette méthode a été utilisée pour évaluer la capacitation des spermatozoïdes dans des programmes de fécondation assistée et aussi pour quantifier les dommages éventuels provoqués par certains traitements de la semence et en particulier par la congélation.

\section{Le noyau : chromati ne / ADN}

La stabilité de la chromatine (ADN + protéines) du spermatozoïde est indispensable à une fécondation normale et au développement embryonnaire. La stabilité de I'ADN dépend des protéines qui y sont associées (protamines et/ou histones). La condensation maximale de la chromatine du noyau des spermatozoïdes éjaculés est liée à la présence des protamines et les défauts de condensation peuvent être à l'origine de certaines formes anormales de la tête.

Un test a été mis au point chez différentes espèces de mammifères domestiques (bovins, porcins et équins) afin d'évaluer la résistance de I'ADN à une dénaturation induite par la chaleur $\left(100^{\circ} \mathrm{C}\right)$ ou par un milieu acide. Cette méthode utilise un colorant fluorescent, l'acridine orange, qui a la propriété d'émettre une fluorescence verte quand il se fixe à de I'ADN natif (double brin), et une fluorescence rouge quand il se fixe à de l'ADN dénaturé (mono brin). Le taux de dénaturation est évalué par le rapport entre la proportion de spermatozoïdes émettant une fluorescence rouge et la population totale de spermatozoïdes (fluorescences verte + rouge). II a été ainsi montré qu'il existait une corrélation négative entre le taux de dénaturation et certains paramètres (mobilité, formes anormales) des spermatozoïdes et avec la fertilité. Ce type d'analyse peut être réalisée en microscopie à fluorescence mais aussi en cytométrie en flux.

\section{Le flagelle}

\section{La membrane plasmi que}

Un test datant des années 70-80 a été mis au point dans l'espèce humaine et consiste à plonger les spermatozoïdes dans une solution hypo-osmotique. En réponse à ce stress, les spermatozoïdes vont réagir comme des osmomètres : si leur membrane plasmique est intacte, ils vont présenter des formes caractéristiques de gonflement au niveau du flagelle correspondant à une entrée d'eau dans la cellule. Si la membrane est lésée, aucun gonflement ne sera observé. Ainsi les spermatozoïdes " gonflés " sont ceux dont la membrane plasmique du flagelle est intacte. Ce test a été utilisé en particulier pour évaluer la qualité des spermatozoïdes équins après congélation.

Les mi tochondries de la pi èce intermédiaire

Les mitochondries sont considérées comme la centrale énergétique du spermatozoïde. Le contenu en énergie peut être évalué par le dosage du contenu en ATP de la cellule et la fonctionnalité des mitochondries par marquage avec une sonde fluorescente, la rhodamine 123. Certains travaux ont mis en évidence une corrélation entre la mobilité des spermatozoïdes et le contenu en énergie des cellules.

La mobilité des spermatozoïdes résulte de l'association de différentes fonctions et en particulier de l'intégrité du flagelle dans son ensemble (membrane, mitochondries, cytosquelette). La mobilité est le critère de qualité le plus utilisé. Cependant son analyse au microscope reste subjective, aussi des systèmes automatisés de mesure assistés par ordinateur ou CASA (Computer Assisted Sperm Analysis) ont été développés. L'analyse des spermatozoïdes se fait grâce à un système optique composé d'une source de lumière, d'un microscope et d'une caméra vidéo qui digitalise les images. Ces images sont ensuite traitées par un ordinateur intégré qui permet de définir de façon objective et répétable des paramètres tels que la proportion de spermatozoïdes mobiles, leur vitesse, la linéarité de leurs trajectoires, etc.

Actuellement tous les éjaculats congelés des étalons nationaux sont sélectionnés sur le critère de mobilité analysé par CASA avant leur mise sur le marché.

\section{Le plasma séminal}

Lors de l'évaluation de la qualité de la semence, il ne faut pas négliger le fluide dans lequel les spermatozoïdes sont émis au moment de l'éjaculation. Le plasma séminal a une importance d'autant plus grande que 1) les spermatozoïdes ne peuvent survivre in vitro dans ce milieu sans être au préalable dilués, 2) sa composition dépend des sécrétions de l'épididyme et des glandes annexes du tractus génital (volume et qualité).

L'utilisation de la spectroscopie de résonance magnétique nous a permis d'identifier et de quantifier des molécules considérées comme 
Figure 1. Profil de spectroscopie de résonance magnétique du proton du plasma séminal équin (Magistrini et al 1995).

Marqueurs des glandes annexes :

- épididyme : carnitine (CAR), glyérophosphorylcholine (GPC)

- ampoule déférente : fonction $N$-acétyl (N-ACET), spermine (SPER), lipoprotéines

- glandes bulbo-uréthrales : acétate (ACET)

- vésicules séminales : citrate $(C / T)$

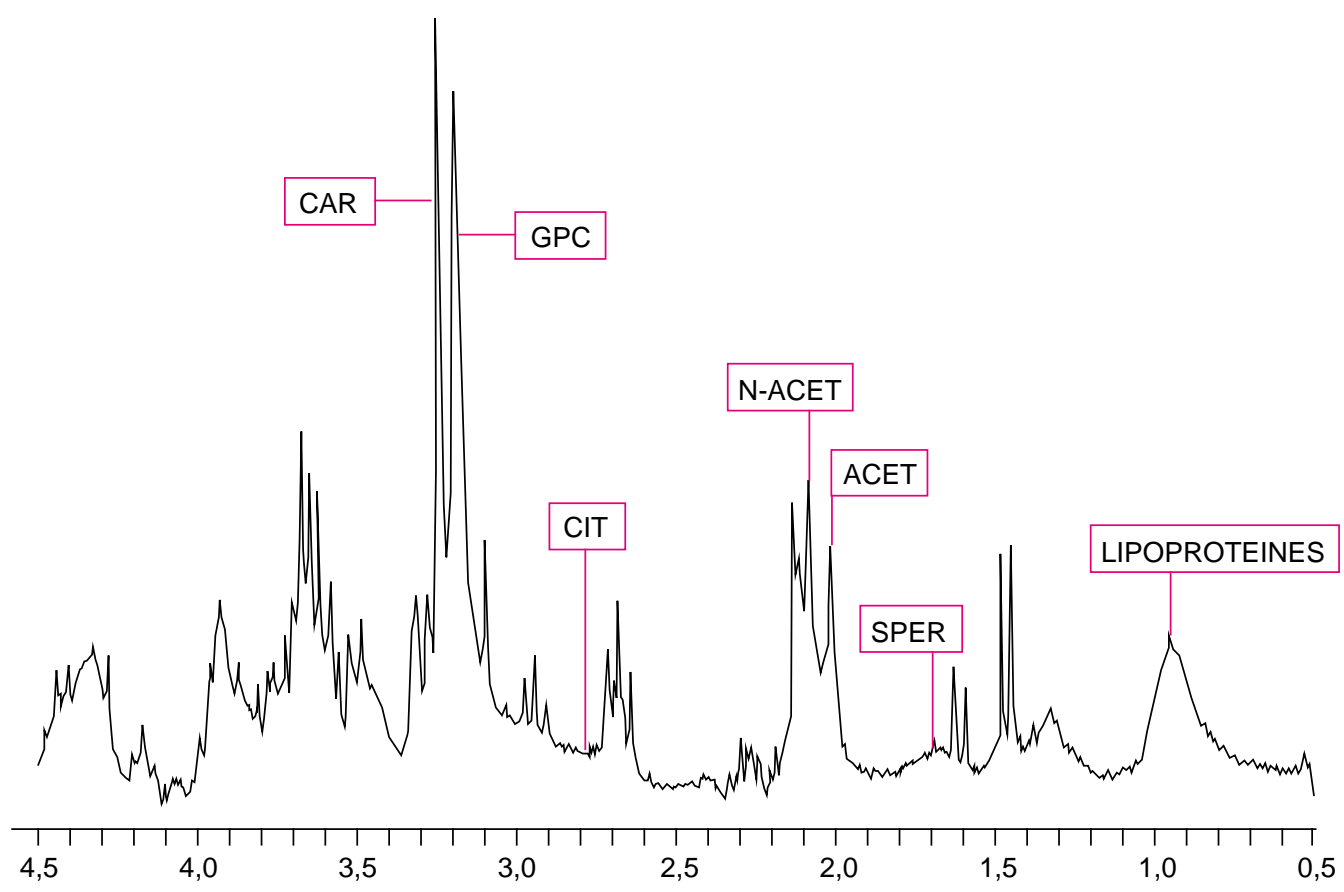

marqueurs des sécrétions de l'épididyme et des glandes annexes du tractus génital. L'intérêt de cette méthode est qu'elle permet en une seule analyse d'évaluer la totalité des molécules alors que la méthode biochimique nécessite de faire autant de dosages qu'il y a de molécules à analyser (figure 1).

Il est donc possible d'estimer la participation de chacune des sécrétions à l'éjaculat. Des relations entre certaines molécules marqueurs et la qualité des spermatozoïdes ont été mises en évidence ; cependant des expériences complémentaires sont nécessaires pour confirmer ces résultats.

\section{Conclusion}

Des corrélations entre différents tests ont été mises en évidence : la mobilité, la viabilité, l'intégrité de l'acrosome, le contenu en ATP, le test HOS; en particulier le test HOS réalisé dès la collecte de la semence est corrélé à la mobilité mesurée après congélation décongélation (Vidament et al 1998). Cependant aucune corrélation n'a pour le moment été mise en évidence entre un test et la fertilité. II est certainement utopique de croire qu'un seul critère pourra caractériser la qualité d'un éjaculat ou la fertilité d'un éta-
Ion. II semble beaucoup plus probable que des associations judicieuses de certains tests nous permettront de progresser dans l'évaluation de la qualité de la semence et par conséquent dans l'estimation de la fertilité de l'étalon.

\section{Pour en savoir plus}

Foucat L., 1990. Principes de base de la résonance magnétique nucléaire. Cahier des techniques de I'INRA, $23,5-24$.

Magistrini M., Guitton E., Le Vern Y., Nicolle J.-C., Vidament M., Kerboeuf D., Palmer E., 1997. New staining methods for sperm evaluation estimated by microscopy and flow cytometry. Theriogenology, 48, 1229-1235.

Magistrini M., 1999. Semen evaluation. In : J uan Samper (ed), Equine breeding management and artificial insemination, $\mathrm{Ch}$. 8. Saunders (sous presse).

Vidament M., Cognard E., Yvon J.M., Sattler M., PalmerE., Magistrini M., 1998. Evaluation of stallion semen before and after freezing. Reprod. Dom. Anim., 33, 271-277.

Malmgren L., 1992. Sperm morphology in stallions in relation to fertility. Acta Veterinaria Scandinavica, Suppl. 88, 39-47. 